\title{
Cardiotoxicity after Additional Administration of Pertuzumab following Long-Term Trastuzumab: Report of 2 Cases
}

\author{
Mayuko Ito $^{a}$ Yoshiya Horimoto $^{a} \quad$ Ritsuko Sasaki $^{a}$ Sakiko Miyazaki ${ }^{b}$ \\ Gotaro Orihata $^{a}$ Mitsue Saito ${ }^{a}$ \\ aDepartment of Breast Oncology, Juntendo University School of Medicine, Tokyo, Japan; \\ bepartment of Cardiology, Juntendo University School of Medicine, Tokyo, Japan
}

\author{
Keywords \\ Breast cancer - Cardiotoxicity · Left ventricular dysfunction · Trastuzumab · Pertuzumab . \\ HER2
}

\begin{abstract}
Pertuzumab, a humanized antibody drug, has improved outcomes of patients with human epidermal growth factor receptor 2 (HER2)-positive breast cancer, when administered in combination with trastuzumab and other chemotherapies. Cardiotoxicity due to trastuzumab is widely recognized, while data on pertuzumab-based treatments in daily clinical practice are lacking. We herein report 2 Japanese patients, aged 72 and 49 years, who developed left ventricular dysfunction after pertuzumab administration, following long-term trastuzumab treatments. Both patients underwent curative surgery for their HER2-positive breast cancer and received anthracycline-based treatments. After developing metastatic disease, trastuzumabbased treatments were administered without cardiac toxicity, but both patients developed left ventricular dysfunction after pertuzumab administration ( 6 and 13 cycles, respectively). Although several large randomized trials have shown no additive effect of pertuzumab on cardiac dysfunction, careful monitoring of cardiac function appears to be necessary in daily practice, particularly for patients with prior long-term trastuzumab treatments.
\end{abstract}

(c) 2021 The Author(s).

Published by S. Karger AG, Basel

\section{Introduction}

Human epidermal growth factor receptor 2 (HER2) protein is overexpressed in $20 \%$ of breast cancer patients and promotes cancer growth. However, the anti-HER2 agent trastuzumab (Tmab), which came on the market early in the 21st century, dramatically improves 
the outcomes of patients with HER2-positive breast cancer [1]. Moreover, the survival of those with HER2-positive metastatic breast cancer (MBC) has been further prolonged with the recent introduction of another anti-HER2 drug, pertuzumab (Pmab) [2].

However, it is widely known that Tmab is cardiotoxic in a certain proportion of patients, and careful monitoring of cardiac function during these treatments is thus recommended. Myocardial cells express HER2 protein and HER2 signals are known to have critical roles in repair of the myocardium [3]. Thus, blockade of HER2 signals might impair or even prevent this process. However, details of the mechanism underlying Tmab-driven heart failure are still largely unknown. Meanwhile, old age, hypertension, diabetes, and previous administration of anthracycline-based chemotherapy are known to be risk factors for Tmab-driven heart failure [4].

Pmab is a monoclonal humanized antibody drug, which binds to an extracellular domain where the HER2 dimer is formed. The binding site is different from that of Tmab, and these 2 drugs can work in a complementary fashion when administered simultaneously [5]. Considering the mechanism of action of Pmab, the addition of Pmab to Tmab likely exacerbates heart failure. In the NeoSphere trial, examining the neoadjuvant setting for early-stage HER2positive breast cancer patients, a slight increase in left ventricular dysfunction (LVD) occurrence was observed in the Pmab group (8\% compared to $2 \%$ of the control group) [6]. However, other major large prospective clinical trials showed no additive effect of Pmab on cardiac dysfunction when combined with Tmab-based treatments [7-9]. Selection bias could exist in prospective clinical trials, i.e., generally, relatively healthy patients are enrolled, while patients who are older tend to have some comorbidities, or must be excluded due to previous treatments, and there are always discrepancies between data from such pivotal clinical trials and routine clinic settings [10]. Hence, we believe that actual clinical data should be accumulated from real-world settings.

We herein report 2 MBC patients who developed severe LVD after Pmab was added to a long-term Tmab regimen.

\section{Case Presentation}

We studied 28 Japanese MBC patients who received Pmab-based treatments during the period from 2014 to 2019 at our department. During the median observation period of 18 months (range: 4-75) from starting Pmab, 2 patients (7.1\%) developed LVD during treatments and both had a history of long-term Tmab administration (Table 1). Reductions in the left ventricular ejection fraction (LVEF) of $10 \%$ or more from baseline to an absolute value of less than $50 \%$ were defined as LVD in this study. There were 16 patients who had previously received Tmab among the 28 patients. The LVD occurrence rate in patients who had previously received Tmab was $12.5 \%$ ( 2 of 16 patients), while none of the 12 patients without prior Tmab developed LVD. Details of the clinical courses of the 2 patients with LVD are presented below.

\section{Case 1}

A 67-year-old woman underwent curative surgery for right breast cancer, and the pathological diagnosis of the primary breast cancer was invasive ductal carcinoma (no special type), high tumor grade and HER2 type (pT2N0M0 stage IIA). As adjuvant systemic treatments, she received anthracycline-based chemotherapy (FEC $75 \mathrm{mg} / \mathrm{m}^{2} \times 4$ cycles), followed by 17 cycles of Tmab. However, she developed lung and liver metastasis 1 year and 9 months postoperatively. Her medical history included hyperlipidemia, but she had no relevant familial history.

\section{Karger'}


Table 1. Details of 2 cases who developed left ventricular dysfunction during Pmab treatment

\begin{tabular}{|c|c|c|c|}
\hline & Case 1 & Case 2 & Non-LVD cases $(n=26)$ \\
\hline Age, years* & 72 & 49 & $63^{* *}($ range: $32-91)$ \\
\hline Organ metastases & Lung, liver, brain & Lung, liver, brain & Lung (15), liver (12), bone (6), others (6) \\
\hline Body mass index & 16.9 & 26.2 & $22.5^{* *}$ (range: $17.3-27.1$ ) \\
\hline Comorbidity & HT & None & HT (4), DM (1), HL (2), other heart disease (2) \\
\hline Total amount of epirubicin, $\mathrm{mg} / \mathrm{m}^{2}$ & 300 & 600 & $153.8^{* *}$ (range: $0-450$ ) \\
\hline \multirow[t]{2}{*}{ Previous Tmab administration } & Yes & Yes & Yes 14/26 (54\%) \\
\hline & & & No $12 / 26(46 \%)$ \\
\hline Total cycles of Tmab & 59 & 38 & $44^{* *}$ (range: $6-132$ ) \\
\hline Total cycles of Pmab & 6 & 13 & $26.4^{* *}$ (range: $6-87$ ) \\
\hline
\end{tabular}

HT, hypertension; DM, diabetes; HL, hyperlipidemia. * At the time when Pmab treatment started. ** Mean values.

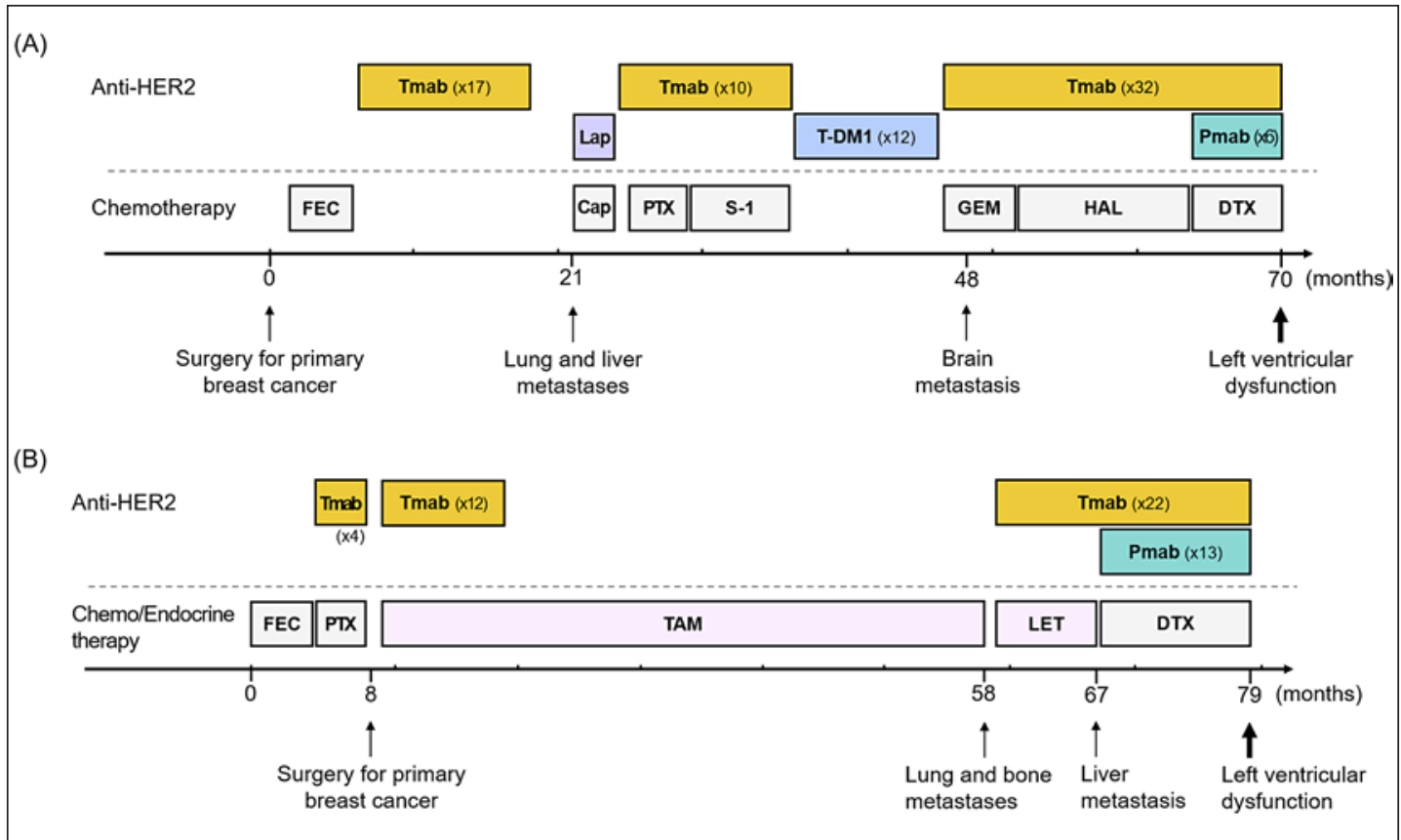

Fig. 1. Clinical course of case 1 (A) and case 2 (B). Lap, lapatinib; Cap, capecitabine; PTX, paclitaxel; S-1, an oral fluoropyrimidine; T-DM1, trastuzumab emtansine; GEM, gemcitabine; HAL, halaven; DTX, docetaxel; TAM, tamoxifen; LET, letrozole.

As MBC treatments, a number of chemotherapeutic regimens combining Tmab and other anti-HER2 agents were administered (Fig. 1A). She had received 53 cycles of Tmab in total before administration of Pmab + Tmab + docetaxel (DTX). After 13 cycles of Pmab + Tmab + DTX, follow-up echocardiography revealed a marked decrease in LVEF (from 70 to 38\%), although she had no symptoms such as a shortness of breath during this regimen. Anti-HER2 treatments were stopped and a $\beta$-blocker and an angiotensin-converting enzyme inhibitor were administered. However, LVEF further dropped to $16 \%$ with an increase in brain natriuretic peptide, and a spironolactone was thus added. Although heart function gradually improved, full recovery took 9 months and her metastatic disease progressed while the anti- 
cancer treatments were suspended. Several chemotherapeutic regimens were then administered without an anti-HER2 reagent, but she died 8 months later.

\section{Case 2}

A 44-year-old woman underwent curative surgery for breast cancer after neoadjuvant chemotherapy (FEC $100 \mathrm{mg} / \mathrm{m}^{2} \times 6$ cycles, followed by 12 cycles of paclitaxel with 4 cycles of Tmab). Pathologically, the histological type was invasive ductal carcinoma (no special type), tumor grade high, and luminal HER2 type (ypT3N1M0 stage IIIA). Additional Tmab (12 cycles) was administered. Endocrine therapy (tamoxifen) was also administered until recurrence. She developed lung and bone metastases approximately 6 years after starting the initial systemic treatment for breast cancer. She had no relevant medical or familial history.

Endocrine therapy (letrozole) in combination with Tmab was first administered (Fig. 1B) for MBC. She had received 25 cycles of Tmab in total before administration of Pmab + Tmab + DTX. After 13 cycles of Pmab + Tmab + DTX, follow-up echocardiography revealed a marked decrease of LVEF (from 71 to 45\%). She had no symptoms specific to LVD. Since her metastases were found to have progressed at the same time, her treatment was changed to another anti-HER2 agent, T-DM1 (Tmab emtansine). No medication was given for the LVD. Despite the switch of systemic therapies, her disease further progressed and she died 4 months later.

\section{Discussion and Conclusion}

Two patients among those who had received Pmab-based treatment for MBC developed LVD, and both had previously received long-term Tmab treatment. Hence, additional Pmab might have directly triggered the LVD development. However, the precise mechanism underlying the onset of LVD remains unknown. It is also possible that the heart disorder caused by anthracycline may have manifested at the time of LVD detection. Indeed, the total doses of anthracycline were high in these 2 patients (epirubicin: 300 and $600 \mathrm{mg} / \mathrm{m}^{2}$, respectively), as compared to the mean dose of $154 \mathrm{mg} / \mathrm{m}^{2}$ in the 26 patients without LVD (non-LVD cases) who received a Pmab-based regimen for MBC but showed no signs of cardiac dysfunction (Table 1). The epirubicin dose was low in the non-LVD cases due mainly to the 15 cases in this group having never received an anthracycline regimen since a Pmab-based regimen had been administered as the early-line therapy for MBC. There were no differences in other factors (age, body mass index, comorbidities, and total dose of Tmab/Pmab), but statistical analysis was not feasible due to the small number of subjects.

LVD developed in $12.5 \%$ of patients who had previously received Tmab, while none of the patients without prior Tmab had LVD. As mentioned above, major prospective randomized studies did not include patients previously treated with anti-HER2 agents. Pmab + Tmab + DTX was the first-line therapy for MBC in the CLEOPATRA study [2, 7], while Pmab-based treatment was the first systemic treatment given to patients in the NeoSphere and APHINITY studies (neoadjuvant and adjuvant settings, respectively) [6, 8]. To assess the safety of additional Pmab, clinical studies, including MBC patients extensively treated with anti-HER drugs, are needed. Nevertheless, LVD might occur with Pmab administration following long-term Tmab, making further careful monitoring of cardiac function necessary.

\section{Acknowledgments}

The authors sincerely acknowledge Dr. Bierta Barfod for language editing.

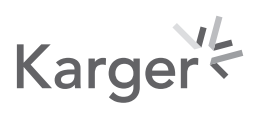




\section{Case Reports in Oncology}

Case Rep Oncol 2021;14:62-66

\begin{tabular}{l|l}
\hline DOI: 10.1159/000513002 & @ 2021 The Author(s). Published by S. Karger AG, Basel
\end{tabular} www.karger.com/cro

Ito et al.: Cardiotoxicity after Additional Administration of Pertuzumab to Trastuzumab

\section{Statement of Ethics}

Written informed consent for the publication of the present case report and any accompanying images was obtained from the patients.

\section{Conflict of Interest Statement}

The authors declare that they have no conflicts of interest to disclose.

\section{Funding Sources}

This research did not receive any specific grant from funding agencies in the public, commercial, or not-for-profit sectors.

\section{Author Contributions}

M.I. and Y.H. contributed to writing of the manuscript. M.I., S.M., G.O., and M.S. were responsible for overall care of the patient. R.S. and M.S. reviewed and edited the manuscript. All authors contributed to discussions and agreed on the final version of the submitted manuscript.

\section{References}

1 Goldhirsch A, Gelber RD, Piccart-Gebhart MJ, de Azambuja E, Procter M, Suter TM, et al. 2 years versus 1 year of adjuvant trastuzumab for HER2-positive breast cancer (HERA): an open-label, randomised controlled trial. Lancet. 2013;382(9897):1021-8.

2 Swain SM, Miles D, Kim SB, Im YH, Im SA, Semiglazov V, et al. Pertuzumab, trastuzumab, and docetaxel for HER2-positive metastatic breast cancer (CLEOPATRA): end-of-study results from a double-blind, randomised, placebo-controlled, phase 3 study. Lancet Oncol. 2020;21(4):519-30.

3 Vermeulen Z, Segers VF, De Keulenaer GW. ErbB2 signaling at the crossing between heart failure and cancer. Basic Res Cardiol. 2016;111(6):60.

4 Jawa Z, Perez RM, Garlie L, Singh M, Qamar R, Khandheria BK, et al. Risk factors of trastuzumab-induced cardiotoxicity in breast cancer: A meta-analysis. Medicine (Baltimore). 2016;95(44):e5195.

5 Baselga J, Cortés J, Kim SB, Im SA, Hegg R, Im YH, et al. Pertuzumab plus trastuzumab plus docetaxel for metastatic breast cancer. N Engl J Med. 2011;366(2):109-19.

6 Gianni L, Pienkowski T, Im YH, Tseng LM, Liu MC, Lluch A, et al. 5-year analysis of neoadjuvant pertuzumab and trastuzumab in patients with locally advanced, inflammatory, or early-stage HER2-positive breast cancer (NeoSphere): a multicentre, open-label, phase 2 randomised trial. Lancet Oncol. 2016;17(6):791-800.

7 Swain SM, Baselga J, Kim SB, Ro J, Semiglazov V, Campone M, et al. Pertuzumab, trastuzumab, and docetaxel in HER2-positive metastatic breast cancer. N Engl J Med. 2015;372(8):724-34.

8 von Minckwitz G, Procter M, de Azambuja E, Zardavas D, Benyunes M, Viale G, et al. Adjuvant pertuzumab and trastuzumab in early HER2-positive breast cancer. N Engl J Med. 2017;377(2):122-31.

9 Shao Z, Pang D, Yang H, Li W, Wang S, Cui S, et al. Efficacy, safety, and tolerability of pertuzumab, trastuzumab, and docetaxel for patients with early or locally advanced ERBB2-positive breast cancer in Asia: the PEONY phase 3 randomized clinical trial. JAMA Oncol. 2020;6(3):e193692-e193692.

10 Brouillette M. The FDA brings real-world data head to head with clinical trials. Nat Med. 2020;26(3):302-3. 